\title{
Interpretation of the modified soil-adjusted vegetation index isolines in red-NIR reflectance space
}

\author{
Zhangyan Jiang $^{\mathrm{a}}$, Alfredo R. Huete ${ }^{\mathrm{a}}$, Jing $\mathrm{Li}^{\mathrm{b}}$, Jiaguo $\mathbf{Q i}^{\mathrm{c}}$ \\ ${ }^{a}$ Department of Soil, Water, and Environmental Science, University of Arizona, Tucson, \\ AZ 85721, USA \\ zjiang@email.arizona.edu (Z. Jiang), ahuete@,Ag.arizona.edu (A. Huete) \\ ${ }^{\mathrm{b}}$ College of Resources Science and Technology, Beijing Normal University, Beijing \\ 100875, China. \\ lijing@,ires.cn \\ ${ }^{\mathrm{c}}$ Department of Geography, Michigan State University, East Lansing, MI 48824, USA \\ qi@msu.edu
}

\begin{abstract}
In red-NIR reflectance space, the Modified Soil Adjusted Vegetation Index (MSAVI) isolines, representing similar vegetation biophysical quantities, are neither convergent to a point nor parallel to each other. Consequently, the treatment of the MSAVI isolines is distinctly different from those of other vegetation index isolines, such as the normalized difference vegetation index (NDVI), the perpendicular vegetation index (PVI), and the soil-adjusted vegetation index (SAVI). In this study, the MSAVI isolines are shown to be the tangent lines of the parabola, (NIR-0.5) ${ }^{2}+2 \mathrm{Red}=0$, and the values of the MSAVI isolines are equal to the ordinates of their tangent points plus 0.5 . These findings provide a graphic interpretation of the MSAVI and are useful in understanding the biophysical characteristics of the MSAVI. The MSAVI isolines are shown to better approximate field data and simulated vegetation biophysical isolines than the other 2-band vegetation index isolines. As the treatment of the MSAVI isolines can be depicted by the parabola curve, the MSAVI can be referred to as a parabola-based vegetation index.
\end{abstract}

Keywords: vegetation indices, MSAVI, vegetation biophysical isolines, tangent lines of a parabola

\section{INTRODUCTION}

Vegetation indices (VIs) derived from satellite remote sensing data are one of the primary sources of information for operational monitoring of the Earth's vegetation cover [1]. Since information contained in a single spectral channel is insufficient to characterize vegetation status, vegetation indices are usually developed to extract vegetation information from two or more spectral bands. The most commonly used VIs utilize the information contained in the red $(\mathrm{R})$ and near infrared $(\mathrm{N})$ reflectances in the form of ratios, such as the normalized difference vegetation index (NDVI)

$$
N D V I=\frac{N-R}{N+R},
$$

or in linear combinations, as in the perpendicular vegetation index (PVI) 


$$
P V I=\frac{N-a R-b}{\left(a^{2}+1\right)^{1 / 2}},
$$

where $a$ and $b$ are the slope and intercept of a soil line, respectively. These VIs have been found to be well correlated with various vegetation biophysical variables such as leaf area index (LAI), fractional vegetation cover, biomass, and the fraction of absorbed photosynthetically active radiation $\left(\mathrm{f}_{\mathrm{APAR}}\right)$, etc. (e.g., [2-8]). An optimal VI should be very sensitive to the desired information (e.g., the amount of vegetation), and as insensitive as possible to perturbing factors such as soil brightness changes or atmospheric variation [9].

Soil background conditions exert considerable influence on partial canopy spectra and the calculated VIs [10]. In order to reduce the soil background effect, Huete [10] proposed using a soil-adjustment factor, $\mathrm{L}$, to account for first-order, vegetation-soil background backscattering, and soil variation, and obtained a soil-adjusted vegetation index (SAVI)

$$
S A V I=\frac{N-R}{N+R+L}(1+L) .
$$

Several modifications have been made to the SAVI equation and the transformed SAVI (TSAVI) [3, 11], modified SAVI (MSAVI) [12], and optimized SAVI (OSAVI) [13] were subsequently proposed. Recently, Gilabert et al. [1] developed a generalized SAVI (GESAVI) based on a simple canopy reflectance model, which takes into account both linear and nonlinear terms related to multiple scattering. Except for the MSAVI, all soil-adjusted VIs have a constant soil-adjustment factor in their equations. The soil-adjustment factor $\mathrm{L}$ in the SAVI equation is 0.5 , factor $\mathrm{X}$ in the TSAVI equation is 0.08 [3], factor $\mathrm{X}$ in the OSAVI equation is 0.16 [13], and factor $\mathrm{Z}$ in the GESAVI equation is 0.35 [1]. Huete [10] found the optimal soil adjustment factor decreased with increases in vegetation amount. So a variable soil adjustment factor could reduce the residual soil noise in the SAVI. The MSAVI was developed with an iterative, variable $\mathrm{L}$ function that becomes

$$
M S A V I=\frac{2 N+1-\sqrt{(2 N+1)^{2}-8(N-R)}}{2} .
$$

It is well recognized that canopy and soil background spectra mix nonlinearly. Whereas the soil-adjusted VIs with a constant soil-adjustment factor treat this nonlinearity with a ratio of reflectances, the MSAVI uses a square root of reflectances.

Soil background influences in VI values for any fixed amount of vegetation can be depicted in red-NIR reflectance space by the discrepancies between VI equation isolines and actual vegetation biophysical isolines $[10,14]$. A VI equation isoline is defined by the line or trace formed by all $\mathrm{R}$ and $\mathrm{N}$ reflectances that result in the same VI value, while a vegetation biophysical isoline is defined as a set of points representing the same optical and structural properties of the canopy, but different soil background brightness conditions [14]. Vegetation biophysical isolines can be derived from reflectances measured over canopies with different soil backgrounds (e.g. $[15,16]$ ), or through canopy reflectances simulated by canopy reflectance models with various input soil backgrounds $[14,17,18]$. The goal of an optimized VI equation or model is for its isolines to approximate vegetation biophysical isolines. The performance of a VI can thus be mostly determined by the accuracy with which the VI isolines predict vegetation biophysical isolines. Understanding the pattern of VI isolines is helpful in understanding the underlying principle or basis of a VI (e.g. how are VI isolines 
distributed and how are their values related), as well as its sensitivity to perturbing factors such as soil background influence.

Furthermore, in order to effectively understand a VI, it is useful to characterize the VI isolines in red-NIR reflectance space, i.e. graphically depict the isolines and establish their relationships to biophysical properties. Ratio-based VIs, such as the NDVI, have isolines convergent to the origin and their VI values can be calculated by the tangent functions of the slope angles of the VI isolines (Fig. 1) [11, 19, 20]. Orthogonal-based VIs such as the PVI have isolines parallel to a soil line and their VI values are determined by the distance between VI isolines and the soil line. Except for the MSAVI, the soil-adjusted VIs mentioned above assume isolines convergent to a single point away from the origin $[1,3,10,11,13]$, and the values of their isolines can be expressed as tangent functions of spectral angles in red-NIR reflectance space $[1,20]$.

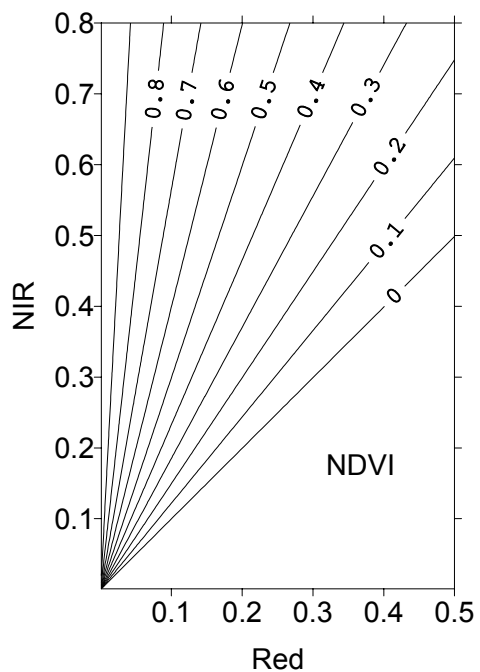

(a)

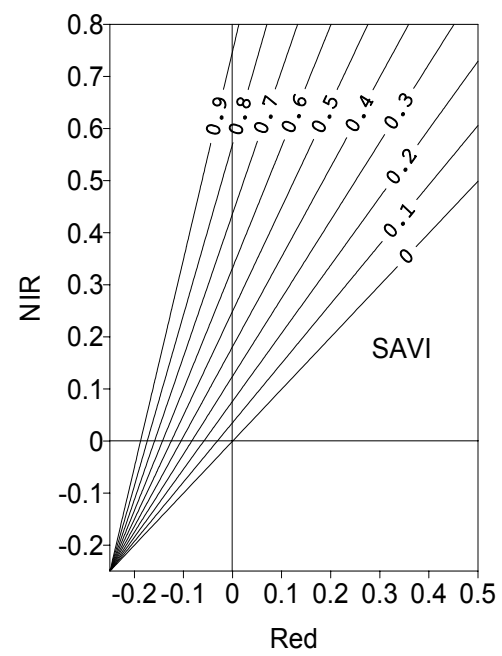

(c)

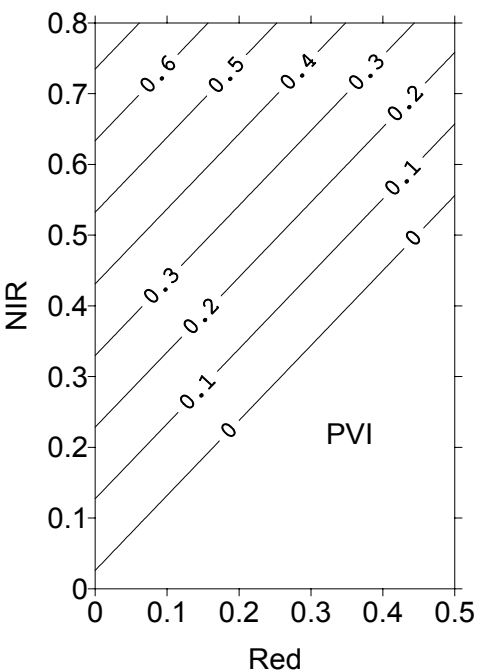

(b)

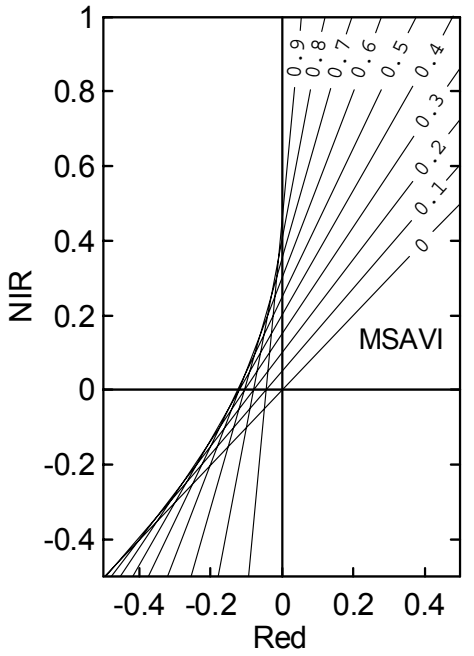

(d)

Fig. 1. Vegetation index isolines in red-NIR reflectance space, (a) NDVI isolines,

(b) PVI isolines, (c) SAVI isolines, (d) MSAVI isolines. 
With a square root term, the MSAVI equation differs substantially from and is more complex than the other VI equations. The MSAVI isolines are neither convergent to a point nor parallel to each other since higher value isolines intersect the soil line more closely to the origin than lower value isolines (Fig. 1d). The treatment of MSAVI isolines and their biophysical relationships are less well studied and not well understood. The purpose of this study is to provide a graphic interpretation of the MSAVI isolines in red-NIR reflectance space and to evaluate the characteristics and properties of the MSAVI isolines.

\section{DERIVATION OF THE MSAVI ISOLINES BASED ON A PARABOLA}

In order to facilitate our analysis, Eq. (4) can be simplified as

$$
M S A V I=N+0.5-\sqrt{(N-0.5)^{2}+2 R},
$$

and the MSAVI isoline equation, i.e., the NIR reflectances expressed as a function of red reflectances, can be resolved from Eq. (5)

$$
N=\frac{1}{1-M S A V I} R+\frac{M S A V I}{2} .
$$

Setting the square root term in Eq. (5) equal to 0, we obtained a parabola in red-NIR reflectance space

$$
(N-0.5)^{2}+2 R=0 .
$$

with the vertex of this parabola at the point $(0,0.5)$ and its opening toward the negative red direction (Fig. 2). A tangent line of this parabola at a point on this parabola $\left(\mathrm{R}_{0}, \mathrm{~N}_{0}\right)$ becomes

$$
N-N_{0}=\frac{2}{1-2 N_{0}}\left(R-R_{0}\right)
$$

Since the point $\left(\mathrm{R}_{0}, \mathrm{~N}_{0}\right)$ is on the parabola, $\mathrm{R}_{0}$ can be expressed as

$$
R_{0}=-\frac{\left(N_{0}-0.5\right)^{2}}{2}
$$

By substituting Eq. (9) into (8), the tangent line of the parabola becomes

$$
N=\frac{1}{1-\left(N_{0}+0.5\right)} R+\frac{N_{0}+0.5}{2} .
$$

$\mathrm{N}_{0}$ can be expressed as a function of $\mathrm{N}$ and $\mathrm{R}$ according to Eq. (10)

$$
N_{0}=N-\sqrt{(N-0.5)^{2}+2 R} .
$$


By comparing Eq. (5) and (11), the relationship between the MSAVI and $\mathrm{N}_{0}$ is

$$
M S A V I=N_{0}+0.5
$$

Substituting Eq. (12) into (10), the tangent line of the parabola, $(\mathrm{N}-0.5)^{2}+2 \mathrm{R}=0$, is found to be the same as Eq. (6), i.e. the MSAVI isoline. Figure 2 shows the MSAVI isolines as the tangent lines of the parabola, and the values of the MSAVI isolines equal to the ordinates of their tangent points plus 0.5 . With increasing slopes of the MSAVI isolines, their tangent points on the parabola shift upward and their MSAVI values increase. Each point with positive red and near infrared reflectances corresponds to an $\mathrm{N}_{0}$ as well as an MSAVI value according to a tangent line of the parabola through this point.

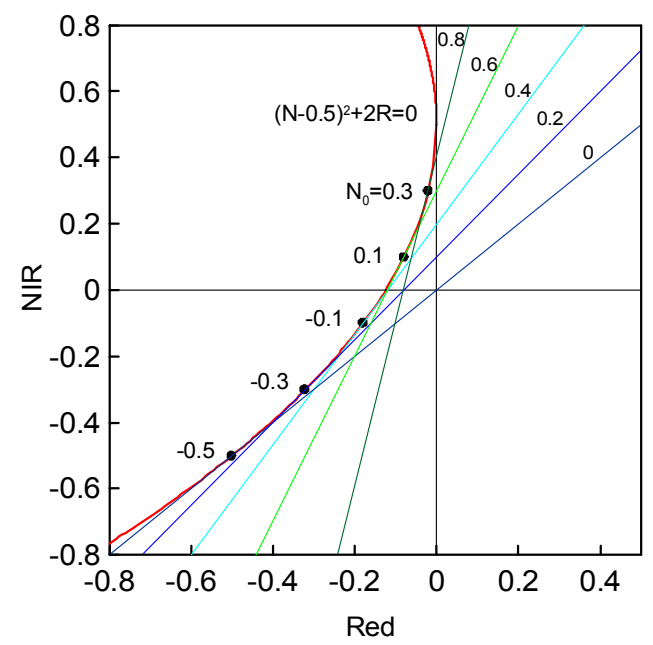

Fig. 2. MSAVI isolines as the tangent lines of a parabola in red-NIR reflectance space.

\section{COMPARISON WITH VEGETATION BIOPHYSICAL ISOLINES}

\subsection{Method and data}

In order to compare the isolines of the NDVI, PVI, SAVI and MSAVI with vegetation biophysical isolines, two ground-measured data sets and a simulated data set were used in this study. The first data set were gathered by Huete et al. [15]. They utilized ground-measured spectral reflectances collected over a series of cotton canopies to study vegetation biophysical isolines in red-NIR reflectance space. Vegetation fraction increases from $0 \%$ to $100 \%$ in a full growing season. At each canopy cover level, soil backgrounds were varied by inserting different soils with a wide range of brightness underneath the vegetation canopies. The soil color ranged from very dark to bright, and the soil moisture varied from wet to dry. The red reflectance of soil background varied between 0.03 and 0.34 . A detailed description of this experiment can be found in [15].

The second data set was measured over a developing corn canopy by Bausch [21]. The procedure of this experiment was similar to the procedure of experiment conduced by Huete et al. [15], but with different vegetation canopies. Spectral reflectances of corn canopies over different soil backgrounds were measured in a nadir view angle at different Leaf Area Index 
(LAI) levels during a growing season. The red reflectance of soil background varied between 0.08 and 0.31 . All the spectral data were collected with similar sun zenith and azimuth angles.

The GeoSail model [22] was used to simulate canopy reflectances, generating the third data set. This model combines the SAIL (scattering from arbitrary inclined leaves) canopy radiative transfer model [23] with the Jasinski canopy geometric model [24-26] to simulate canopy spectral reflectances for discontinuous tree canopies. The SAIL model provides the within-tree radiative transfer calculations and Jasinski's model combines the SAIL results into a scene reflectance. Scene reflectance is determined in the model by calculating an areaweighted average of three landscape components: illuminated canopy, illuminated background, and shadowed background.

The input parameters to the GeoSail model are listed in Table 1. Leaf optical properties and sun zenith angle were followed as [22]. A soil line, NIR=1.062Red +0.026 , is assumed and the red reflectance of the soil background was varied between 0.05 and 0.37 . A planophile leaf angle distribution function is used and the shape of canopies is cylinder. As the trees grow, they become taller and wider to occupy space within the canopy [22]. Thus, the vegetation fraction (i.e. fraction area covered by tree canopies) increases with the increase of leaf area index (LAI). A nonlinear relationship between vegetation fraction, $f$, and LAI is assumed as

$$
f=1-\exp (-0.522 L A I) \text {. }
$$

Table 1. Input parameters used in the GeoSail model.

\begin{tabular}{ll}
\hline Parameters & Values \\
\hline Red reflectance and transmittance of leaf & 0.07 and 0.032 \\
NIR reflectance and transmittance of leaf & 0.505 and 0.407 \\
Leaf angle distribution & Planophile \\
Soil line & NIR=1.062Red +0.026 \\
Red reflectance of soil background & $0.05-0.37$ \\
Sun zenith angle & $42^{\circ}$ \\
Height to width ratio of canopies & 1 \\
Shape of canopies & Cylinder \\
\hline
\end{tabular}

\subsection{Results}

The vegetation biophysical isolines for the three canopies are presented in Fig. 3. The isoline for $0 \%$ vegetation cover is the bare soil line (Fig. 3a, 3c). The biophysical isoline of the corn canopy with least LAI (0.004) approximate the bare soil, given the negligible quantity of plants [21]. For the three canopy cases, increases in the vegetation fraction or LAI resulted in vegetation biophysical isolines that increased in slope, decreased in length, and shifted upward in the positive NIR and negative red directions. The decreases in isoline 'length' are associated with a declining influence of the soil background with increases in vegetation cover.

The biophysical isolines simulated from the GeoSail model are not straight lines and a small curvature can be observed at intermediate vegetation levels. However, these biophysical isolines can be approximated by straight lines fairly accurately, with $\mathrm{R}^{2}>0.99$. Yoshioka et al. $[14,17]$ derived linear vegetation biophyscial isolines by separating the first-order interactions of spectra between vegetation canopy and its soil background from higher-order interactions, and found the contribution of the higher-order interactions were less than 5\%.

VI isoilnes do not overlap biophysical isolines at all vegetation density levels, resulting in soil background effects on VIs. The discrepancy between a biophysical isoline and a VI isoline can be represented by the angles between the biophysical isoline and the VI isoline 
across the mid-point of each vegetation fraction (or LAI) isoline, calculated by the VI isoline slope angle minus the biophysical isoline slope angle (Fig. 4).

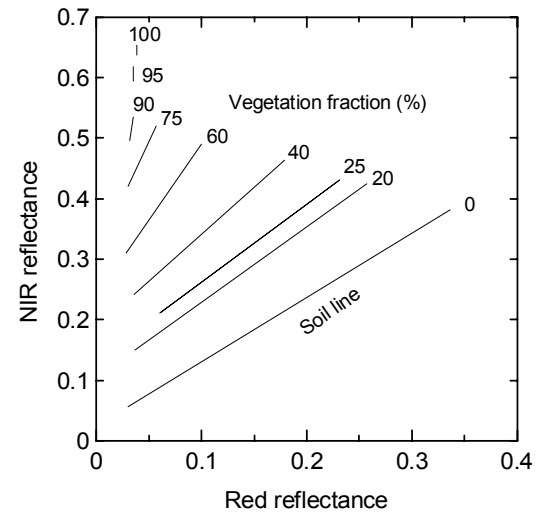

(a)

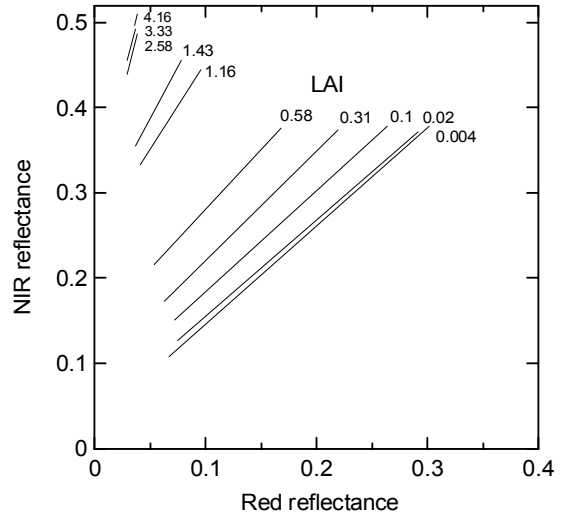

(b)

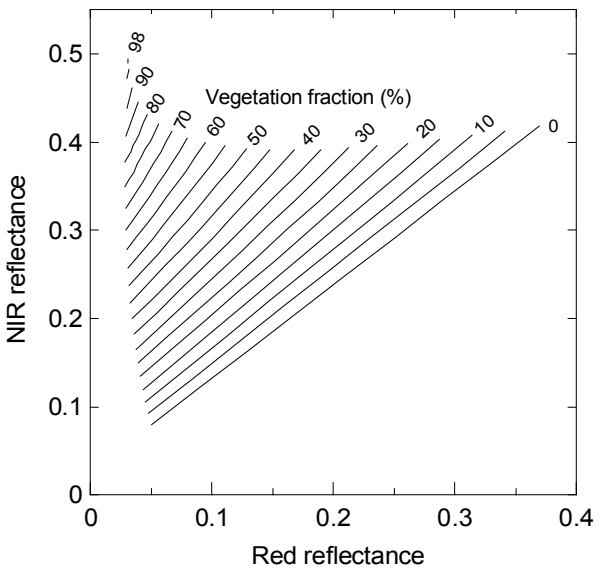

(c)

Fig. 3. Vegetation biophysical isolines at different vegetation density (vegetation fraction or LAI) levels. (a) cotton biophysical isolines (Huete et al., 1985), (b) corn

biophysical isolines (Bausch, 1993), (c) simulated tree biophysical isolines.

For the cotton canopy, the NDVI isolines deviated significantly from the biophysical isolines at vegetation fractions less than $75 \%$, with smaller deviations at higher vegetation fractions (Fig. 4a). The largest discrepancy occurred at $40 \%$ vegetation fraction. Positive deviations indicate slope angles of NDVI isolines are larger than those of vegetation biophysical isolines. The angles between the PVI isolines and vegetation biophysical isolines became greater with increases of vegetation fraction and the slope angles of biophysical isolines. Because the PVI isolines are parallel to the soil line, slope angles of biophysical isolines are larger than those of PVI isolines, particularly at high vegetation density levels. The SAVI produced small and positive angle differences when vegetation fractions are below $40 \%$, and produced negative angle differences when vegetation fractions are more than $60 \%$. In contrast, the angle differences produced by the MSAVI were smaller than those of the SAVI at all vegetation fractions.

Similar angle differences were found over the corn canopy and the simulated tree canopy for the four VIs (Fig. 4b, 4c). The NDVI produced significant, positive angle differences. The largest difference occurred when LAI is about 1 for corn canopies, and when vegetation 
fraction is $55 \%$ for the simulated canopies. Increasing angle differences produced by the PVI were also observed with increases in vegetation density. The angle differences for SAVI and MSAVI switched from positive to negative as vegetation amount increases, but the differences remained small. The angle differences for MSAVI were the smallest over the full range of vegetation density levels, indicating that the MSAVI isolines matched the vegetation biophysical isolines the best.

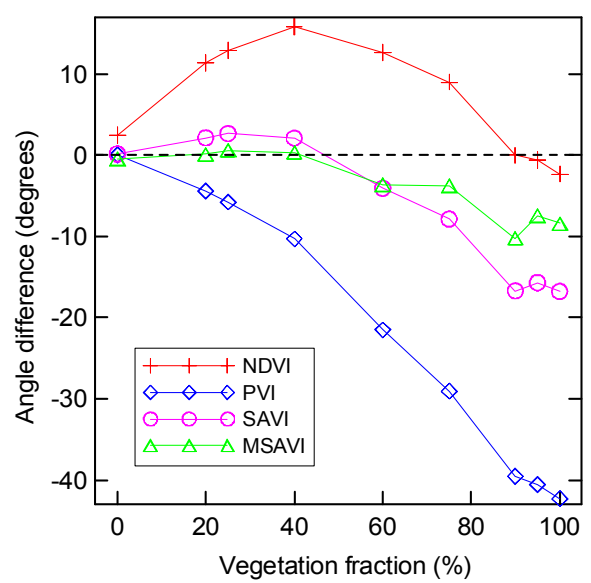

(a)

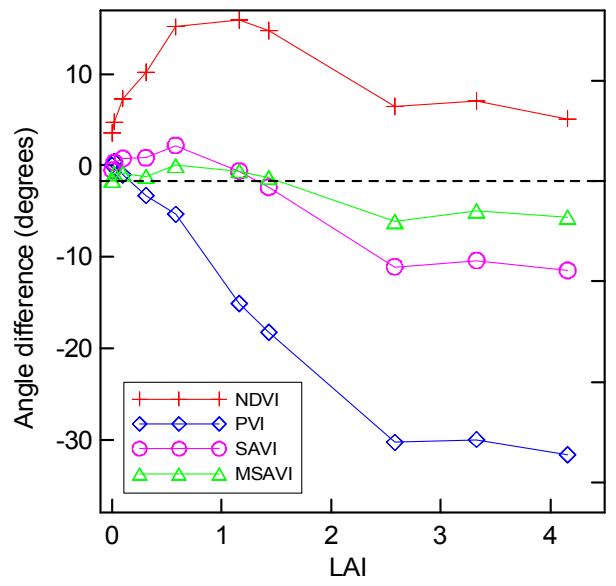

(b)

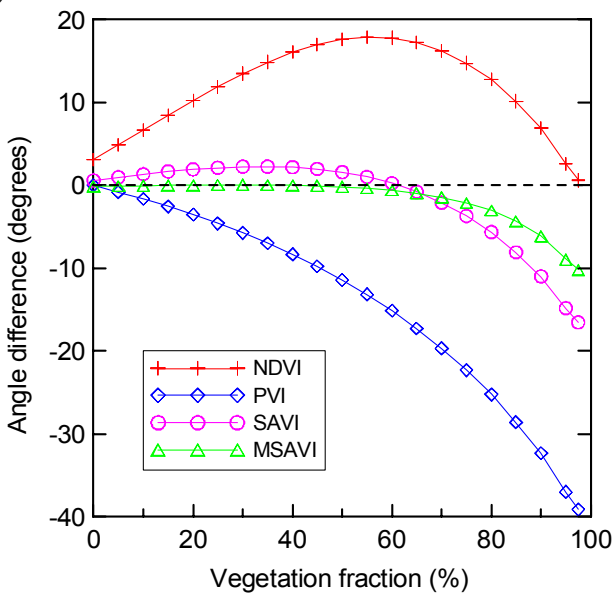

(c)

Fig. 4. Angles between vegetation biophysical isolines and VI isolines across the mid-points of vegetation fraction (or LAI) isolines, (the VI isoline slope angle minus the biophysical isoline slope angle) (a) cotton canopy, (b) corn canopy, (c) simulated tree canopy.

In order to compare soil influences in the four VIs, a relative soil noise value for each vegetation density level was defined as

$$
\text { Relative soil noise }=\left(V I_{d a r k}-V I_{\text {bright }}\right) /\left(V I_{\max }-V I_{\min }\right),
$$

where $\mathrm{VI}_{\text {dark }}$ and $\mathrm{VI}_{\text {bright }}$ are the $\mathrm{VI}$ values for constant vegetation canopies over the darkest and brightest soil backgrounds used in the experiments, and $\mathrm{VI}_{\max }$ and $\mathrm{VI}_{\min }$ are the maximum and minimum VI values for each data set, respectively. The NDVI produced significant soil 
noise when vegetation fractions were less than $60 \%$ or LAI was less than 1.5 , and was maximum at vegetation fractions between $20 \%$ and $40 \%$ or LAI between 0.5 and 1 (Fig. 5). The positive noise indicates NDVI overestimated vegetation amount over dark soil background.

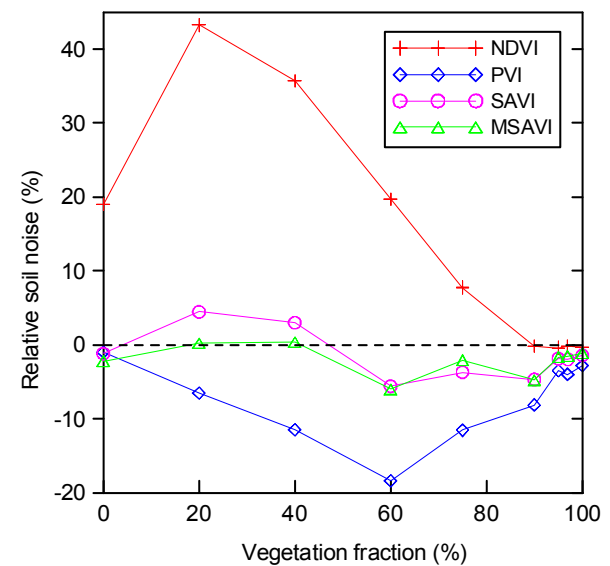

(a)

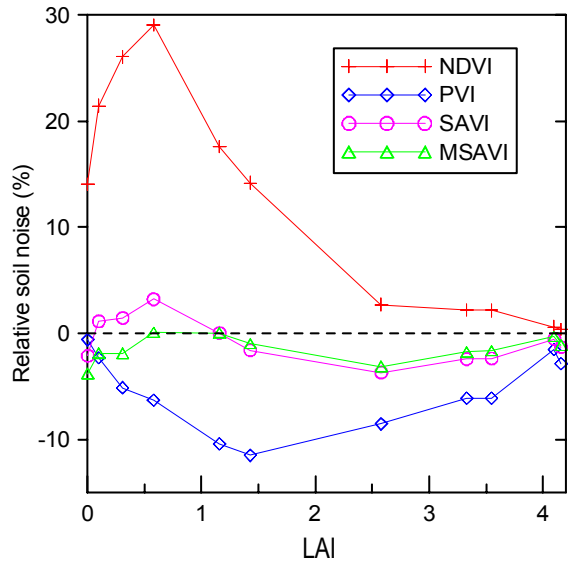

(b)

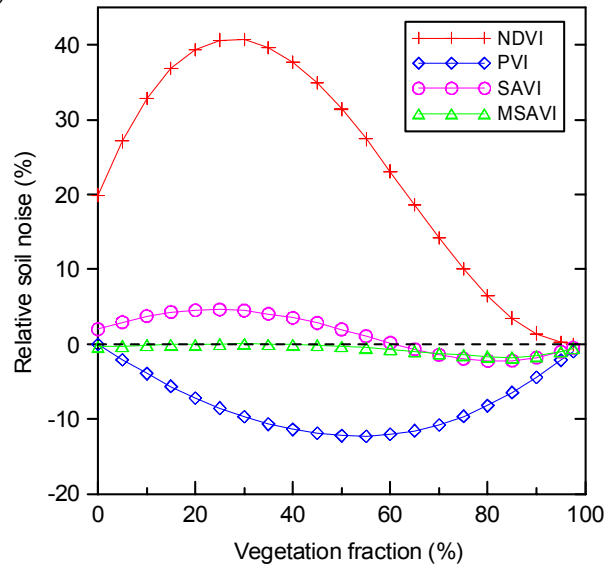

(c)

Fig. 5. Relative soil noise in the four VIs at various vegetation fractions or LAI levels over (a) cotton canopy, (b) corn canopy, (c) simulated tree canopy.

The PVI produced significant soil noise at intermediate vegetation levels, not at the highest density levels, because the lengths of the vegetation biophysical isolines at high fractional covers or LAI became very small (Fig. 3), i.e. soil background produced insignificant effects on canopy reflectances at high vegetation levels, even though the angle differences between PVI isolines and biophysical isolines are greatest at highest vegetation density. Soil noise of a VI is the synergistic result of angle difference between the VI isolines with the biophysical isolines and the lengths of the biophysical isolines. Negative soil noise indicates the PVI underestimated vegetation amount over dark soil background.

The SAVI and MSAVI produced small positive soil noise at low vegetation levels and small negative soil noise at high vegetation levels. The soil noise of the MSAVI was smallest among the three canopy cases. 


\section{CONCLUSION}

In red-NIR reflectance space, the treatment of the MSAVI isolines was shown to be distinctly different from the isoline patterns of the other VIs. The treatment of the MSAVI isolines can be graphically determined by a parabola curve with the location of the vertex and curvature of the parabola influencing the arrangement of the isolines. The MSAVI isolines were determined to be the tangent lines of the parabola, $(\mathrm{N}-0.5)^{2}+2 \mathrm{R}=0$, with the values of the MSAVI isolines equal to the ordinates of their tangent points plus 0.5 . In this sense, the MSAVI can be referred to as a parabola-based VI.

These findings provide a graphic interpretation of the MSAVI for an improved understanding of the biophysical characteristics of the MSAVI. The most significant difference between the MSAVI isolines and other soil-adjusted VIs is that the MSAVI isolines are not necessarily convergent to a single point, resulting in more flexibility and better agreement with vegetation biophysical isolines, and further reducing soil background influences. We found MSAVI isolines to closely approximate field-measured and modeled canopy biophysical isolines over differing canopy structures and a wide range vegetation fraction, LAI, and soil conditions, indicating the robust nature of the MSAVI.

Further studies encompassing different vegetation canopies would be beneficial to more completely evaluate the performance of these VIs. The data sets used in this study were at the ground level without consideration of atmospheric effects. Studies evaluating atmospheric and bidirectional (view and sun angle) effects on the MSAVI biophysical isolines, such as those reported by Huete and Liu [27], will improve our understanding of performance of these indices.

\section{Acknowledgments}

We thank Dr. Bausch for providing the corn canopy data. This work was funded by MODIS contract NNG04HZ20C (A. Huete).

\section{References}

[1] M. A. Gilabert, J. González-Piqueras, F. J. García-Haro, and J. Meliá, “A generalized soil-adjusted vegetation index," Remote Sens. Environ. 82, 303-310 (2002) [doi:10.1016/S0034-4257(02)00048-2].

[2] K. F. Huemmrich, "The GeoSail model: a simple addition to the SAIL model to describe discontinuous reflectance," Remote Sens. Environ. 75, 423-431 (2001) [doi:10.1016/S0034-4257(00)00184-X].

[3] F. Baret, and G. Guyot, "Potentials and limits of vegetation indices for LAI and APAR assessment," Remote Sens. Environ. 35, 161-173 (1991) [doi:10.1016/00344257(91)90009-U].

[4] G. Asrar, E. T. Kanemasu, and M. Yoshida, "Estimates of leaf area index from spectral reflectance of wheat under different cultural practices and solar angle," Remote Sens. Environ, 17, 1-11 (1985) [doi:10.1016/0034-4257(85)90108-7].

[5] A. J. Richardson, C. L. Weigand, D. F. Wajura, D. Dusek, and J. L. Steiner, "Multisite analyses of spectral -biophysical data for sorghum," Remote Sens. Environ. 41, 71-82 (1992) [doi:10.1016/0034-4257(92)90062-O].

[6] T. N. Carlson, and D. A. Ripley, "On the relation between NDVI, fractional vegetation cover, and leaf area index," Remote Sens. Environ. 62, 241-252 (1997) [doi:10.1016/S0034-4257(97)00104-1].

[7] Z. Jiang, A. R. Huete, J. Chen, Y. Chen, J. Li, G. Yan, and X. Zhang, "Analysis of NDVI and Scaled Difference Vegetation Index Retrievals of Vegetation Fraction," Remote Sens. Environ 101, 366-378 (2006) [doi:10.1016/j.rse.2006.01.003]. 
[8] J. G. P. W. Clevers, "The application of a weighted infrared-red vegetation index for estimating leaf area index by correction for soil moisture," Remote Sens. Environ. 29, 25-37 (1989) [doi:10.1016/0034-4257(89)90076-X].

[9] M. M. Verstraete and B. Pinty. "Designing optimal spectral indexes for remote sensing applications," IEEE Trans. Geosci. Remote Sens. 34, 1254-1265 (1996) [doi:10.1109/36.536541].

[10] A. R. Huete, "A soil-adjusted vegetation index (SAVI)," Remote Sens. Environ. 25, 295-309 (1988) [doi:10.1016/0034-4257(88)90106-X].

[11]F. Baret, G. Guyot, and D. Major, "TSAVI: a vegetation index which minimizes soil brightness effects on LAI and APAR estimation," in $12^{\text {th }}$ Canadian symposium on remote sensing and IGARSS'90, Vancouver, Canada, 10-14 July 1989, 1355-1358, (1989).

[12] J. Qi, A. Chehbouni, A. R. Huete, H. Y. Kerr, and S. Sorooshian, "A modified soil adjusted vegetation index," Remote Sens. Environ. 48, 119-126 (1994) [doi:10.1016/0034-4257(94)90134-1].

[13] G. Rondeaux, and F. Baret, "Optimization of soil-induced vegetation indices." Remote Sens. Environ 55, 95-107 (1996) [doi:10.1016/0034-4257(95)00186-7].

[14] H. Yoshioka, T, Miura, A. R. Huete, and B. G. Ganapol, "Analysis of vegetation isolines in red-NIR reflectance space," Remote Sens. Environ. 74, 313-326 (2000) [doi:10.1016/S0034-4257(00)00130-9].

[15] A. R. Huete, R. D. Jackson, and D. F. Post, "Spectral response of a plant canopy with different soil backgrounds," Remote Sens. Environ. 17, $37-53$ (1985) [doi:10.1016/0034-4257(85)90111-7].

[16] B. M. Díaz and G. A. Blackburn, "Remote sensing of mangrove biophysical properties: from a laboratory simulation of the possible effects of background variation on spectral vegetation indices," Int. J. of Remote sens. 24, $53-73$ (2003) [doi:10.1080/01431160305012].

[17] H. Yoshioka, A. R. Huete, and T. Miura, "Derivation of vegetation isoline equations in the red-NIR reflectance space," IEEE Trans. Geosci. Remote Sens 38, 838-848 (2000) [doi:10.1109/36.842012].

[18] H. Yoshioka, "vegetation isoline equations for an atmosphere-canopy-soil system," IEEE Trans. Geosci. Remote Sens. 42, 166-175 (2004) [doi:10.1109/TGRS.2003.817793].

[19] C. Ünsalan, and K. L. Boyer, "Linearized vegetation indices based on a formal statistical framework," IEEE Trans. Geosci. Remote Sens. 42, 1575-1585 (2004) [doi:10.1109/TGRS.2004.826787].

[20] Z. Jiang, A. R. Huete, J. Li, Y. Chen, "An analysis of angle-based with ratio-based vegetation indices," IEEE Trans. Geosci. Remote Sens. 44, 2506-2513 (2006) [doi:10.1109/TGRS.2006.873205].

[21] W. Bausch, "Soil background effects on reflectance-based crop coefficients for corn," Remote Sens. Environ. 46, 213-222 (1993) [doi:10.1016/00344257(93)90096-G].

[22] K. F. Huemmrich, "The GeoSail model: a simple addition to the SAIL model to describe discontinuous canopy reflectance," Remote Sens. Environ. 75, 423-431 (2001) [doi:10.1016/S0034-4257(00)00184-X].

[23] W. Verhoef, "Light scattering by leaf layers with application to canopy reflectance modeling: the SAIL model," Remote Sens. Environ. 16, 125- 141 (1984) [doi:10.1016/0034-4257(84)90057-9].

[24] M. F. Jasinski, "Functional relation among subpixel canopy cover, ground shadow, and illuminated ground at large sampling scales," In: Society of photo optical instrumentation engineers. Orlando, FL, (1990). 
[25] M. F. Jasinski, and P. S. Eagleson, "The structure of red-infrared scattergrams of semivegetated landscapes," IEEE Trans. Geosci. Remote Sens. 27, 441-451 (1989) [doi:10.1109/36.29564].

[26] M. F. Jasinski, and P. S. Eagleson, "Estimation of subpixel vegetation cover using red-infrared scattergrams," IEEE Trans. Geosci. Remote Sens. 28, 253-267 (1990) [doi:10.1109/36.46705].

[27] A. R. Huete, and H. Liu, "An error and sensitivity analysis of the atmospheric- and soil-correcting variants of the NDVI for the MODIS-EOS," IEEE Trans. Geosci. Remote Sens. 32, 897-905 (1994) [doi:10.1109/36.298018].

Zhangyan Jiang is a post-doctor in the Department of Soil, Water, and Environmental Science at the University of Arizona. He received his BS from the Jiangxi Normal University, Nanchang, China, and his MS and PhD degrees from Beijing Normal University, Beijing, China, all in geography, in 1998, 2002 and 2006 respectively. His research interests include optimization of remotely sensed vegetation indices, and retrieval of vegetation biophysical parameters.

Alfredo Huete is a professor in the Department of Soil, Water and Environmental Science at the University of Arizona, Tucson, Arizona USA where he serves as present chair of the interdisciplinary academic program, Committee on Remote Sensing and Spatial Analysis. He received his MS from the University of California at Berkeley and $\mathrm{PhD}$ from the University of Arizona in 1984, under the guidance of Ray D. Jackson. He is a MODIS Science Team Member and is responsible for the development, implementation, and validation of the vegetation index products. He is part of the NPOESS Preparatory Project Science Team to evaluate vegetation indices as environmental data records (EDRs). He is also an active member of the Large-Scale Biosphere Atmosphere in the Amazon (LBA) interdisciplinary experiment and serves on the Editorial review board for Remote Sensing of Environment.

Jing $\mathbf{L i}$ is a professor in the College of Resource Science and Technology at Beijing Normal University, Beijing, China. He received the BS degree in geography and MS degree in graphics and remote sensing both from Peking University, Beijing, China in 1982 and 1985 respectively. His research interests include, remote sensing of natural resource, remote sensing applications in natural disaster reduction, monitoring of oceanic and coastal ecology by remote sensing.

Jiaguo Qi is the director of the Center for Global Change and Earth Observations and an associate professor of geography at Michigan State University. His research interests focus on theoretical development and applications of remote sensing technologies to study the dynamics of the Earth's surface and its environmental impacts at variable spatial and temporal scales. He has published 58 scientific articles, 56 refereed proceedings articles, 3 book chapters and 1 book. He has a BS in physics from Harbin Teacher's Normal University in China. He completed his master's and doctorate degrees in soil and water science at the University of Arizona. 